\title{
Un estudio general sobre colisiones peatonales y su fatalidad en Ciudad de México durante los años 2004 a 2015
}

(1) Ramiro Flores-Xolocotzi

Comisión de Desarrollo y Bienestar Social, Senado de la República, México. https://orcid.org/oooo-0001-9979-1057

Recibido: 20 de agosto de 2019. Aceptado: 9 de junio de 2020.

\begin{abstract}
Resumen
Se realizó un análisis estadístico sobre colisiones peatonales y su fatalidad (peatones muertos frente a peatones heridos) por atropellamientos en vialidades de zonas urbanas de Ciudad de México en el período 2004 a 2015. Igualmente, se estimaron un modelo de Regresión Binomial Negativa Truncada y un modelo Logit Binomial para analizar el efecto de algunas variables sociodemográficas, ambientales, de tiempo y lugar sobre la frecuencia de colisiones peatonales y la probabilidad de fatalidad en atropellamientos. Los resultados permiten concluir que variables como el día de la semana (lunes a domingo); horario (mañana, tarde, noche y madrugada); lugar de la colisión (intersección y no intersección); tipo de vehículo involucrado; causa atribuible de la colisión (peatón, conductor u otra); además del sexo, edad y presencia de aliento alcohólico del conductor, junto con la posible fuga de este último: influyen de manera diferenciada sobre las variables respuesta de los modelos estimados (conteo de colisiones y probabilidad de la fatalidad). Futuras investigaciones deberán estudiar particular y detalladamente las relaciones estadísticas encontradas entre las variables estudiadas. Finalmente, los resultados de esta investigación pretenden con todas sus limitantes ser complementarias a estudios e investigaciones de índole geográfica, de ingeniería del transporte y de salud pública.
\end{abstract}

Palabras clave: Accidentes de tránsito. Ciudades caminables. Calles y avenidas. Logit. Regresión Binomial Negativa Truncada.

\section{A general study about pedestrian collisions and their fatality in Mexico City during the years 2004 to 2015}

\begin{abstract}
A statistical analysis was carried out about pedestrian collisions and their fatality (dead pedestrians versus injured pedestrians) due to run over in urban areas of Mexico City in the period 2004 to 2015. Likewise, a Truncated Negative Binomial Regression model
\end{abstract}


and a Binomial Logit model were estimated to analyze the effect of some sociodemographic, environmental, time and place variables on the frequency of pedestrian collisions and the probability of fatality in collisions. The results allow us to conclude that variables such as the day of the week (Monday to Sunday); schedule (morning, afternoon, night and dawn); place of collision (intersection and non-intersection); type of vehicle involved; attributable cause of the collision (pedestrian, driver or other); in addition with sex, age and presence of alcoholic breath of the driver, together with the possible escape of the driver, they influence in the response variables of the estimated models differently (collision count and probability of collision fatality). Future research should study in particular and in detail the statistical relationships found among the variables studied. Finally, the results of this research are intended with all its limitations to be complementary to studies and research of a geographic type, transport engineering and public health.

Keywords: Traffic accidents. Walkable cities. Streets and avenues. Logit. Truncated Negative Binomial Regression.

Palavras-chave: Acidentes de trânsito. Cidades caminháveis. Ruas e avenidas. Logit. Regressão binomial negativa truncada.

\section{Introducción}

Las vías públicas ${ }^{1}$ de circulación en una ciudad forman parte de los espacios públicos que constituyen una red junto con parques, plazas y otros elementos urbanos (Mumford, 1981; Jacobs, 2011; Speck, 2012; Espinosa, 2013; Gehl, 2016). Bajo el enfoque de Jacobs (2011) y Gehl (2016) el actor social que camina por esos espacios públicos, al que se llamará peatón ${ }^{2}$, es fundamental en la revitalización y animosidad de los espacios públicos citadinos.

Dicho pensamiento ha cobrado relevancia en ciudades europeas, estadounidenses e iberoamericanas, en el sentido de la necesidad de empoderar al peatón en los espacios públicos de los que hace uso (Southworth, 2005; Lo, 2009; Isaksson-Hellman, 2012; Talen y Koschinsky, 2013; Forsyth, 2015; Dovey y Pakfka, 2019). Sin embargo, el peatón puede ser un actor vulnerable al interaccionar en las vías de transporte con vehículos (automóviles, camionetas, camiones, motocicletas, bicicletas y en algunos casos trenes y tractores). Lo anterior es debido a que la interacción puede generar accidentes de tránsito en donde el peatón, es el actor más vulnerable a ser herido o fallecer durante la colisión (Baker et al., 1974; Van Houten, 2011; Gopalakrishnan, 2012; Speck, 2012; Hernández-Hernández y De Haro-De León, 2014; Mohan, 2019).

De esta forma, son fundamentales el estudio y análisis de los accidentes vehiculares con peatones (atropellamientos o colisiones peatonales) para proveer medidas que prevengan los atropellamientos peatonales, su mortalidad y lesiones (Kröyer, 2015; Zhou et al., 2016; Cantillo et al., 2016; Gehl, 2016; Rafiemanzelat et al., 2017; Mohan, 2019. Lo anterior favorecería el empoderamiento de los peatones en los espacios públicos. En consecuencia, las colisiones peatonales deberían formar parte de un marco teórico social particular de la planeación urbana en cuestiones de movilidad (Vargas y Carrillo, 2009; Gopalakrishnan, 2012; Talen y Koschinsky, 2013; Loo y Anderson, 2015; Patterson et al., 2018; Canitez, 2019). Por lo anterior, este trabajo busca estudiar y analizar los atropellamientos bajo el marco teórico que se expone a continuación.

1 En este trabajo se entenderá vía pública de circulación como las calles, caminos, avenidas o autopistas (entendidas los cuatro tipos como vialidades) que son utilizadas para movilizar personas y/o bienes (Pérez y Gómez, 2014). 


\section{Antecedentes y marco teórico}

Las colisiones peatonales son particularmente de interés en áreas médicas, al considerarse un problema de salud pública. Se tiene que la Organización Mundial de la Salud (OMS, 2015) reporta al año 2013 una tasa mundial de mortalidad en accidentes de tránsito de 17,4 por cada 100 mil habitantes. Para los países del continente americano la tasa es de15,9 decesos por cada 100 mil habitantes cifra por abajo del máximo que se presenta en el continente africano que es de 26,6. Además, considerando el total de personas fallecidas en accidentes de tránsito en 2013, se reporta un 22\% de peatones fallecidos a nivel mundial y un porcentaje similar para el continente americano. Estando el máximo en el continente africano con un 39\%. Un análisis detallado de estas cifras se puede consultar en OMS (2015).

Pérez y Gómez (2014), con datos del Instituto Nacional de Estadística y Geografía (INEGI) mencionan para México que, en el año 2012, se registró una tasa de 354,85 colisiones de tránsito por cada 100 mil habitantes. Citan también una tasa de lesionados de 150 por cada 100 mil habitantes en zonas urbanas. Igualmente, Pérez y Gómez (2014) señalan que en 2012 del total de lesionados 53\% fueron conductores o pasajeros; $23 \%$ motociclistas; $13 \%$ peatones y $12 \%$ ciclistas. De acuerdo con Hernández-Hernández y De Haro-De León (2014), en México la tasa de mortalidad por siniestros viales es de las más altas del mundo. Mientras que en México hay una tasa de 20,7 muertes por cada mil habitantes, países como Suiza y Japón tienen una tasa inferior a 5. Para Ciudad de México (en adelante CDMX) el Instituto de Investigaciones Parlamentarias (IIP, 2012), señala que en 2011 se registraron: 16.466 accidentes de tránsito anuales. Además, reporta un total de 976 y 149 peatones heridos y muertos respectivamente para el mismo año.

En un marco teórico de movilidad urbana, abordar el problema de atropellamientos, no sólo es un problema de salud, sino que se puede extender a un análisis social en planeación urbana al ser la vía pública un servicio primordial en propuestas de provisión y gestión de espacios públicos (Tabasso, 2012; Lindenau y Böhler-Baedeker, 2014; Rafiemanzelat et al., 2017; Carlotta y Betolini, 2017; Papadimitriou et al., 2017; Shinar, 2017; Berjisian y Habibian, 2019; Canitez, 2019; Dada et al., 2019; Shatu et al., 2019).

En consecuencia, se han desarrollado estudios que describen y analizan al peatón y al conductor del vehículo involucrados en accidentes de tránsito y atropellamientos (Helmer, 2015; Shinar, 2017; Hall et al., 2018; Dovey y Pafka, 2019). Sobre el tema, Elvik (2006) señala que la investigación empírica ha determinado un gran número de factores o variables de riesgo que están estadísticamente asociados con accidentes en vías públicas de circulación y cuya presencia incrementa la probabilidad de una colisión. Al respecto, Loo y Anderson (2015) señalan que los factores de riesgo forman parte de una "teoría de causalidad de colisiones en vías públicas". Sobre esta teoría, Elvik (2006), menciona que pueden existir innumerables factores/variables de riesgo que pueden ser causa de accidentes en vialidades, pero sus efectos pueden desplegar notables regularidades. Tales regularidades son denominadas por Elvik (2006) como leyes de causalidad de accidentes de las cuales el autor propone cuatro:

1. Ley universal de aprendizaje. Señala que la capacidad de detectar y controlar los peligros del tráfico aumenta uniformemente a medida que se incrementa el número de uso de las vías de circulación.

2. Ley de eventos raros. Establece que cuanto más raro sea un factor de riesgo, mayor será su efecto sobre la tasa de accidentes.

3. Ley de complejidad. Describe que a mayor cantidad de información por tiempo que la persona tenga que atender, mayor será la probabilidad de que cometa un error en la vía de circulación. 
4. Ley de la capacidad cognitiva. Establece que cuanto más se acerca la capacidad cognitiva de la persona a sus límites, mayor es la tasa de accidentes.

Dentro de la teoría de causalidad, Elvik (2006) reconoce elementos como la propensión individual a sufrir accidentes, los errores humanos y deseos personales por el riesgo. Estos aspectos conductuales individuales no se profundizarán en esta investigación, sin embargo, pueden revisarse en Wilde (1982), Shaoman y Ying, (2015) y Shinar (2017).

Para abordar el análisis de la fatalidad ${ }^{3}$ del accidente de tránsito, se puede recurrir al enfoque de "sistema seguro", el cual considera al sistema vial de manera holística considerando la interacción entre usuarios, vías públicas, vehículos y velocidades de circulación (Loo y Anderson, 2015). Bajo esta visión, Bliss y Breen (2009) han desarrollado el "modelo teórico de manejo de seguridad de vialidades" que considera un "sistema de responsabilidad de tres fases" y cuyo objetivo final es eliminar la mortalidad y lesiones por accidentes. Las fases del sistema son:

» Funciones de manejo institucional. Comprende un marco legal, el monitoreo y evaluación de la seguridad en vialidades y la coordinación entre instituciones. Esta función conduce a intervenciones.

》 Intervenciones. Comprende aspectos de planeación, diseño, operación y uso de vialidades; flujo de vehículos en vialidad y medidas de atención/rehabilitación de las víctimas de accidentes. Esta fase conduce a resultados.

» Resultados. Obtiene resultados de las fases anteriores que deben ser evaluados a través de costos sociales que miden el éxito/fracaso de las fases anteriores.

De esta forma el estudio de los atropellamientos peatonales bajo un análisis de "teoría de causalidad" y de la perspectiva del "sistema de responsabilidad", permite conocer y analizar respectivamente diversos factores causales de la colisión peatonal (en adelante $\mathrm{CP}$ ) y los riesgos de mortalidad del accidente (Hijar, 2000; Rodríguez-Hernández et al., 2011; Gopalakrishnan, 2012; Hernández-Hernández y De Haro-De León, 2014; Quistberg et al., 2014; Hernández-Vásquez et al., 2016). Consecuentemente, diversos estudios han estimado modelos de conteo de colisiones (Poisson y Binomial Negativa) y de riesgo (modelos Logit y Probit) para estudiar y analizar lo anterior (Guerrero, 2015; Helmer, 2015; Hinojosa et al., 2015; Kröyer, 2015; Zhou et al., 2016; Cantillo et al., 2016; Haule et al. 2019; Wang et al., 2019).

Los estudios citados toman en cuenta aspectos sociales como los relacionados con la responsabilidad causal del peatón y del conductor involucrado en las CP. Igualmente el marco teórico de causalidad de atropellamientos, podría considerar también variables como:

» Perfil sociodemográfico del peatón y conductor.

» Estado físico y mental de los actores sería el caso de alguna discapacidad física/ mental.

》 Lugar del accidente (intersección/no intersección).

» Consumo de alcohol por parte del conductor/peatón.

"Variables temporales como hora y día del accidente.

»Variables ambientales/climáticas como temperatura y lluvia que pueden afectar la percepción, la capacidad de procesamiento de información de conductores/peatones y el desplazamiento del vehículo. Por lo tanto, pudiesen incrementar el número de colisiones y su fatalidad (Wanvik, 2009; Zhang et al., 2013). 
» Tipo de vehículo involucrado (automóvil, camión, motocicleta, otros) y su velocidad.

» Presencia de semáforos, pasos de cebra (marcas en el pavimento de intersecciones que indican prioridad peatonal) y otras señalizaciones en vías de circulación. Estas pueden ser consultadas en Sanz (2016).

Bajo estas perspectivas teóricas, en consecuencia, es necesario señalar las limitantes de esta investigación. Lo anterior, en el sentido de que los modelos estimados en este trabajo no tienen un enfoque predictivo de colisiones y riesgo de muerte sino más bien son de tipo estimativo para identificar algunos factores que inciden en las $\mathrm{CP}$ y en la fatalidad de las colisiones. Además, esta investigación no es un análisis conductual, espacial, social y económico, de tipo integral y detallado del peatón y del conductor. La investigación tampoco comprende una revisión detallada del análisis del entorno urbano que incluya variables como alumbrado público, condición del pavimento y señalizaciones viales que puedan afectar la incidencia de las CP y el riesgo de mortalidad.

Por lo anterior, existen otros enfoques teóricos que, si bien no son desarrollados en este trabajo, futuras investigaciones podrían considerarlos a profundidad. Son ejemplo de ello, teorías de la conducta de conductores y peatones basados en el enfoque de racionalidad limitada de Herbert Simon o en la teoría de la prospectiva de Kahneman y Tversky respectivamente. Ambos enfoques desarrollados en psicología y economía conductual, se han aplicado en accidentes de tránsito y colisiones peatonales (SchmidtDaffy, 2014; Shaoman y Ying, 2015; Chen, 2016; Shinar, 2017). Sin olvidar, perspectivas clásicas como la teoría de la homeostasis del riesgo aplicadas a estudiar las decisiones del conductor (Wilde, 1982).

Considerando lo anterior, este trabajo busca analizar de manera general, el efecto que tienen algunas variables o factores causales de tipo sociodemográfico, temporal, de lugar y ambiental en las CP y su fatalidad peatonal (en adelante FP), teniendo como objetivos particulares:

» Estimar y analizar estadísticas de colisiones de vehículos de transporte con peatones y estadísticas de peatones muertos y peatones heridos en accidentes de tránsito en zonas urbanas de Ciudad de México durante el período 2004 a 2015. Para ello se consideró el día (lunes a domingo), horario (madrugada, mañana, tarde y noche y lugar del accidente en intersección o no intersección (la intersección es como tal la vialidad que cruza o "interseca" con otra).

" Estimar un modelo de conteo de CP y un modelo de FP por colisión de tipo peatonal (hubo o no hubo muertos en la colisión) en accidentes de tránsito para zonas urbanas de Ciudad de México durante el período 2004 a 20015.

» Analizar el número de colisiones y la probabilidad de FP en función de: a) variables sociodemográficas (edad y sexo del conductor); b) sociales (causa atribuible del atropellamiento y aliento alcohólico del conductor); c) temporales del accidente (horario, período semanal y año); d) tipo de vehículos involucrados; c) lugar del atropellamiento (intersección(/no intersección) y d) variables ambientales (precipitación pluvial promedio trimestral y temperatura promedio trimestral).

La hipótesis general de trabajo sustenta que las variables demográficas, sociales, temporales, ambientales, de lugar y tipo de vehículos involucradas en los accidentes, influyen significativamente en la frecuencia y fatalidad de las colisiones peatonales. 


\section{Caracterización del área de estudio}

La CDMX, al año 2015, es una urbe con 8.918.653,00 habitantes que colinda al norte, este y oeste con Estado de México y al sur con el estado de Morelos (INEGI, 2017). De acuerdo a INEGI (2019a), 99,5\% de la población es urbana y el 0,5\% es rural, con una densidad poblacional de 5.967 personas por $\mathrm{km}^{2}$. Según INEGI (2019a), en el 87\% del territorio de CDMX el clima es templado subhúmedo; en un 7\% es seco y semiseco y en un $6 \%$ es templado húmedo. En cuanto a temperatura, en los meses de marzo a mayo se presentan temperaturas mayores a $25^{\circ} \mathrm{C}$ y las más bajas se presentan en el mes de enero y fluctúan alrededor de $5^{\circ} \mathrm{C}$. Con respecto a lluvias, en CDMX en sus regiones secas son de alrededor de $600 \mathrm{~mm}$ anuales y en las zonas templadas húmedas llegan alrededor de $1200 \mathrm{~mm}$ anuales. En la Figura 1, se puede observar un mapa con la división política de CDMX, constituida por 16 alcaldías referidas en el mapa de acuerdo con INEGI (2019b).

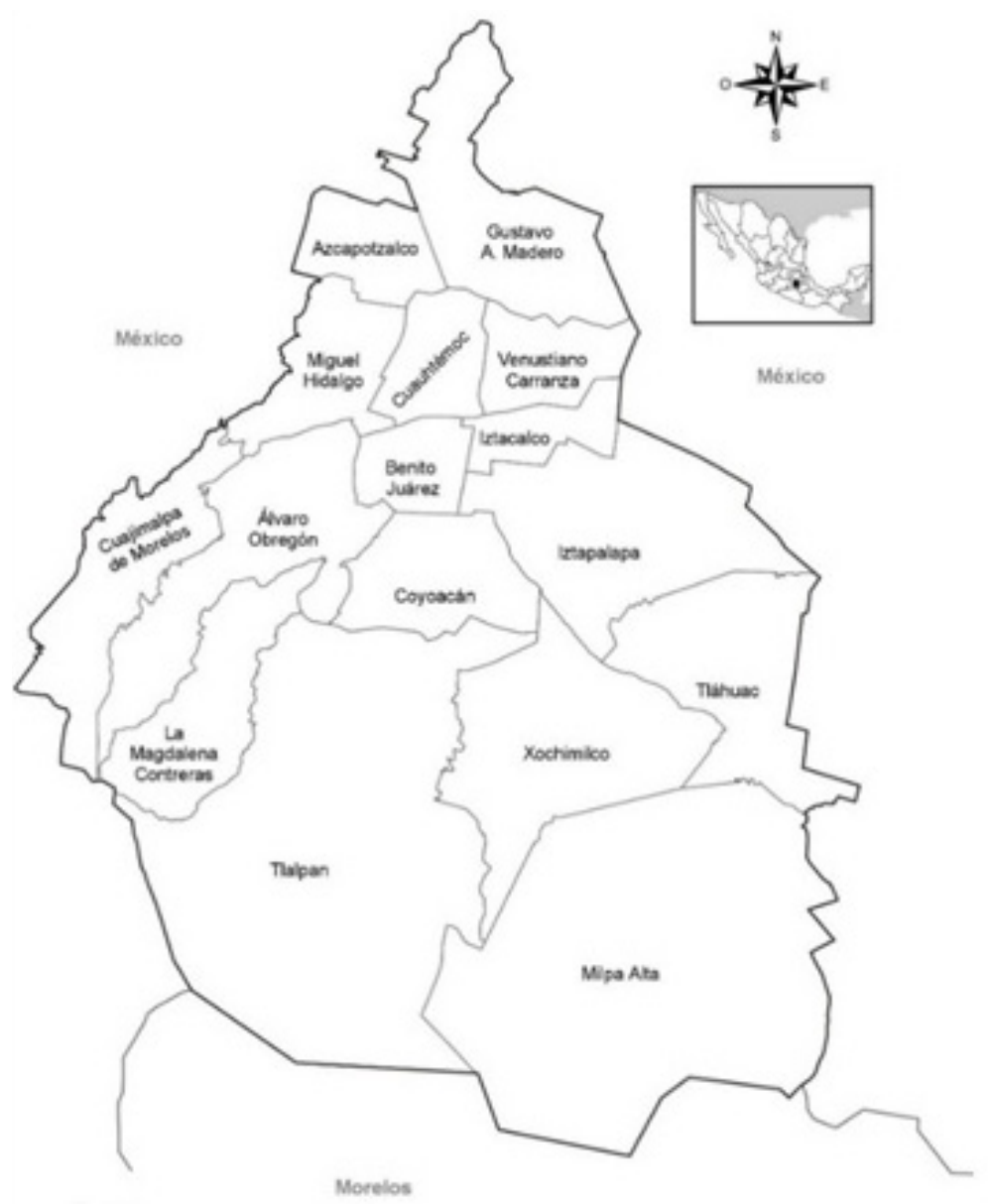

Figura 1. División política de CDMX. Fuente: INEGI (2019).

En cuanto a colisiones peatonales en CDMX, en el período de 2004 a 2015 se acumula un total de 12.324 colisiones, con un total de 1.738 peatones muertos y 11.437 peatones heridos, con valores promedio de 1.027, 145 y 953 respectivamente para los indicadores señalados (INEGI, 2017). El desglose de resultados para dicho período se encuentra en el Cuadro 1. 
Cuadro 1. Estadísticas de CP en CDMX. Fuente: elaboración propia con datos de INEGI (2020).

\begin{tabular}{|l|c|c|c|}
\hline Año & $\begin{array}{c}\text { Número de Colisiones de } \\
\text { Tipo Peatonal }\end{array}$ & $\begin{array}{c}\text { Número de peatones } \\
\text { heridos en CP }\end{array}$ & $\begin{array}{c}\text { Número de peatones } \\
\text { muertos en CP }\end{array}$ \\
\hline 2004 & 1.049 & 996 & 115 \\
\hline 2005 & 948 & 879 & 137 \\
\hline 2006 & 1.051 & 953 & 165 \\
\hline 2007 & 1.015 & 940 & 161 \\
\hline 2008 & 1.010 & 943 & 127 \\
\hline 2009 & 1.027 & 961 & 133 \\
\hline 2010 & 1.091 & 1.012 & 164 \\
\hline 2011 & 1.030 & 951 & 147 \\
\hline 2012 & 1.248 & 1.154 & 171 \\
\hline 2013 & 1.106 & 1017 & 176 \\
\hline 2014 & 1.010 & 924 & 150 \\
\hline 2015 & 739 & 707 & 92 \\
\hline
\end{tabular}

\section{Metodología}

Bajo el enfoque de la "teoría de causalidad" y del "sistema de responsabilidad", esta investigación adopta una metodología estadística para analizar algunos factores relacionados con las $\mathrm{CP}$ y su fatalidad en zonas urbanas de CDMX, tal como se describirá posteriormente.

En una etapa previa a la metodología se seleccionaron las variables causales. Para esto, se partió en un primer paso, de las variables enlistadas previamente en el marco teórico de causalidad y del sistema de responsabilidad. A partir de esa lista se obtuvieron algunas variables que son consideradas por el Instituto Nacional de Estadística y Geografía (en adelante INEGI) para la elaboración de sus estadísticas anuales de accidentes de tránsito terrestre en zonas urbanas y suburbanas (INEGI, 2016). En consecuencia, las variables a ser seleccionadas, estuvieron limitadas por la información y registros que tiene disponible INEGI anualmente de 2004 a 2015 (INEGI, 2020).

Las variables dependientes comprendieron, el número de atropellamientos. Esta es provista por INEGI (2020) en forma de colisiones peatonales reportadas en Ciudad de México. La variable fatalidad peatonal se construyó a partir del número de peatones heridos y muertos por colisión también reportado por INEGI (2020).

\section{Elaboración de bases de datos}

Identificadas las variables dependientes e independientes se presentó la necesidad de elaborar dos bases de datos: una constituida por información anual de colisiones peatonales y la segunda por información anual de fatalidad peatonal. Las dos bases comprendieron: solo eventos ocurridos de 2004 a 2015 en zonas urbanas de CDMX siguiendo la clasificación de INEGI (2020) que separa los accidentes de tráfico en zonas urbanas y zonas no urbanas. Esta actividad se realizó durante diciembre de 2017 a enero de 2018, considerando datos e información que tiene INEGI (2020) y que son de libre acceso para su consulta a través de Internet.

Los datos anuales se obtuvieron inicialmente en formato "DBF". Posteriormente se exportaron a hoja de cálculo de Excel para cada año. A partir de las bases de datos 
anuales obtenidas, estas se depuraron y se ordenaron considerando el trimestre anual en que ocurrió el accidente (diciembre-febrero, marzo-mayo, junio-agosto, septiembrenoviembre). Los trimestres coinciden aproximadamente con los períodos estacionales de invierno, primavera, verano y otoño respectivamente del año correspondiente.

Para ordenar las bases de datos, se empleó el software SPSS versión 22, para obtener una base de datos con el número de CP de 2004 a 2015 en CDMX a nivel anual ( $\mathrm{n}=9,218$ observaciones) y una segunda base de datos sobre FP (peatones heridos y muertos) por CP durante el mismo período ( $\mathrm{n}=12,324$ observaciones).

También, es necesario destacar que se añadieron a las bases de datos (de CP y de RP), las variables de temperatura promedio trimestral y precipitación promedio trimestral, ambas del trimestre cuando ocurrió el accidente, Las bases de datos de temperatura y lluvia se obtuvieron de Comisión Nacional del Agua (CONAGUA, 2018).

Cada base de datos estuvo constituida por una variable dependiente (Cuadro 2) y un conjunto de variables independientes descritas en el Cuadro 3. La estimación de modelos de regresión, permitió identificar para cada uno, un conjunto de variables de interacción que se encuentran en el Cuadro 4 . Las bases de datos elaboradas se emplearon, respectivamente, para la estimación de un Modelo de CP (del tipo Regresión Binomial Negativa Truncada, en adelante RBNT) y un Modelo de FP (Regresión Logística Binaria) respectivamente.

Cuadro 2. Dos variables dependientes que constituyeron la base de datos y que se emplearon respectivamente en el modelo de CPy en el Modelo de FP. Fuente: Elaboración propia con datos e información de INEGI (2020).

\begin{tabular}{|c|c|c|c|}
\hline \multicolumn{2}{|c|}{$\begin{array}{c}\text { Modelo de CP (Regresión Binomial Negativa } \\
\text { Truncada) }\end{array}$} & \multicolumn{2}{|c|}{ Modelo de FP (Regresión Logística Binaria) } \\
\hline Variable & Escala de Medición & Variable & $\begin{array}{l}\text { Escala de } \\
\text { Medición }\end{array}$ \\
\hline $\begin{array}{c}\text { Yi. } \\
\text { Número de colisiones. }\end{array}$ & $\begin{array}{c}\text { Cuantitativa } \\
\text { (variable discreta de } \\
\text { conteo). }\end{array}$ & $\begin{array}{c}\text { Yi. } \\
\text { o: Sólo hubo peatón/peatones } \\
\text { heridos en la CP. } \\
\text { 1: Hubo al menos un peatón } \\
\text { muerto en la CP. }\end{array}$ & $\begin{array}{c}\text { Nominal } \\
\text { (Dicotómica) }\end{array}$ \\
\hline
\end{tabular}

Cuadro 3. Variables independientes consideradas en los modelos estimados. Fuente: Elaboración propia con datos e información de INEGI (2020). Los datos climatológicos se obtuvieron de CONAGUA (2018).

Día de la semana: lunes (BASE), martes, miércoles, jueves, viernes, sábado y domingo.

7 Variables dicotómicas nominales:

o: No.

1: Sí.

Hora del día: madrugada (12 a.m. a 5 a.m.) (BASE); mañana (6 a.m. a 11 a.m.); tarde (12 p.m. a 5

p.m.) y noche (6 p.m. a 11 p.m.).

Cuatro variables dicotómicas nominales

o: No.

1: Sí.

Lugar de la colisión.

Variable dicotómica nominal:

o: En no intersección.

1: En intersección (esquina).

Causa atribuible del accidente: Conductor (BASE); peatón; otra (falla del vehículo, mala condición del camino, otra no especificada).

Tres variables dicotómicas nominales:

o: No.

1: Sí. 
Vehículo involucrado: automóvil.

Variable dicotómica nominal:

o: Sí.

1: No.

Vehículo involucrado: camioneta, microbús, camión urbano de pasajeros, ómnibus, trolebús, camioneta de carga, camión de carga y tractor.

Variable dicotómica nominal:

o: No.

1: Sí.

Vehículo involucrado: motocicleta.

Variable dicotómica nominal:

o: No.

1: Sí.

Vehículo involucrado: bicicleta, ferrocarril u otro no especificado.

Variable dicotómica nominal:

o: No.

1: Sí.

Sexo del conductor: mujer (BASE), hombre, no se sabe porque se fugó.

Tres variables dicotómicas nominales:

o: No.

1: Sí.

Aliento alcohólico del conductor: No se sabe porque se fugó o no se registró (BASE); sin aliento alcohólico; con aliento alcohólico.

Tres variables dicotómicas nominales:

o: No.

1: Sí.

Edad del Conductor: $\leq 25$ años (BASE); Entre >25 y $\leq 50$ años; >50 años; no se sabe porque se fugó o no se registró.

Cuatro variables dicotómicas nominales:

o: No.

1: Sí.

Precipitación pluvial promedio en milímetros, del trimestre en que ocurrió el accidente: diciembre-febrero, marzo-mayo, junio-agosto y septiembre-noviembre.

Variable cuantitativa

Temperatura promedio en grados centígrados del trimestre en que ocurrió el accidente: diciembre-febrero, marzo-mayo, junio-agosto, septiembre-noviembre.

Variable cuantitativa

Año: 2004 (BASE), 2005, 2006, 2007, 2008, 2009, 2010, 2011, 2012, 2013, 2014, 2015

Doce variables dicotómicas:

o: No.

1: Sí.

Cuadro 4. Variables de interacción consideradas en los modelos estimados. Fuente: Elaboración propia con datos e información de INEGI (2020).

\begin{tabular}{llll}
\hline \multicolumn{1}{c}{$\begin{array}{c}\text { Modelo de CP (Regresión Binomial } \\
\text { Negativa Truncada) } \\
\mathrm{n}=9,218\end{array}$} & \multicolumn{1}{c}{ Modelo de FP (Regresión Logit Binomial) } \\
$\mathrm{n=12,324}$
\end{tabular}




\begin{tabular}{|c|c|c|c|}
\hline $\begin{array}{l}\text { Interacción: } \\
\text { (sábado)*(noche). }\end{array}$ & $\begin{array}{l}\text { Variable } \\
\text { dicotómica: } \\
\text { o: No } \\
\text { 1: Sí }\end{array}$ & $\begin{array}{l}\text { Interacción: } \\
\text { (Automóvil) *(Sin } \\
\text { aliento alcohólico). }\end{array}$ & $\begin{array}{l}\text { Variable dicotómica: } \\
\text { o: No. } \\
\text { 1: Sí. }\end{array}$ \\
\hline \multirow{2}{*}{\multicolumn{2}{|c|}{$\begin{array}{l}\text { Interacción: (Motocicleta)*(Hombre)*(Entre } \\
>25 \text { y<=50 años). }\end{array}$}} & $\begin{array}{l}\text { Interacción: } \\
\text { (Automóvil)* } \\
\text { (Precipitación pluvial } \\
\text { promedio trimestral). }\end{array}$ & $\begin{array}{l}\text { Variable dicotómica: } \\
\text { o: No. } \\
\text { 1: Sí. }\end{array}$ \\
\hline & & $\begin{array}{l}\text { Variable dicotómica: } \\
\text { o: No. } \\
\text { 1: Sí. }\end{array}$ & \\
\hline
\end{tabular}

Además, se realizó una consulta a INEGI (2019c) a través de la solicitud CAS-11118Y8R5Z7 CRM:0874347. La respuesta a la solicitud permitió determinar que en la variable causa atribuible del accidente en su categoría "peatón o pasajero", se reduce sólo a peatón en colisiones peatonales. Manteniéndose tal cual las otras categorías de causa atribuibles del accidente que son: conductor del vehículo, falla del vehículo, mala condición del camino u otra no especificada.

\section{Análisis estadístico general}

En una primera etapa de la investigación, se realizó un análisis cualitativo estadístico en el cual se estimó el conteo (frecuencia) de $\mathrm{CP}$ considerando el día (lunes a domingo), horario (madrugada, mañana, tarde y noche) y lugar del accidente (intersección y no intersección de la vialidad) durante el período 2004 a 2015 en zonas urbanas de CDMX. Lo anterior es tal como se realizó en otros estudios de accidentes peatonales (Baker et al., 1974; Gopalakrishnan, 2012; Hernández-Hernández y De Haro-De león, 2014; Quistberg et al., 2014; Kröyer, 2015; Hal et al., 2018) . Lo mismo se realizó para analizar el número de heridos y muertos en función del día, horario y lugar de la CP. En esta fase se estimaron promedios y totales para describir dichas relaciones. Estas estimaciones se realizaron en el software SPSS versión 22.

\section{Estimación de modelos}

\section{Modelo de colisión peatonal}

Posteriormente, se estimó el modelo de CP. Por la naturaleza de la variable dependiente (conteo), se optó por una RBNT tipo NB-2 truncada en cero. Lo anterior debido a que la base de datos comprende sólo colisiones reportadas, consecuentemente se excluye la posibilidad de cero colisiones de la base de datos (Liu et al., 2013; Hilbe, 2014; Scott y Freese 2014; Shonkwiler, 2016). Para comprender este modelo se tiene que considerar lo siguiente:

» Los modelos de Regresión Binomial Negativa, pueden estimar de manera eficiente modelos en donde la variable dependiente posea una varianza que tienda a ser mayor que su media (aspecto conocido como sobredispersión) (Hilbe, 2011; Hilbe, 2014; Liu et al., 2014).

»En el modelo de Regresión Binomial Negativa, el tipo NB2 indica que siendo la media, entonces la varianza será . De esta manera el modelo a estimar, tendría la siguiente función de verosimilitud $\left(L_{N B 2}\right)$ de acuerdo a Hilbe $(2011,2014)$, Liu et al. (2013) y Scott y Freese (2014):

》 Considerando que la función se trunca en $\mathrm{Yi}=0$, entonces el modelo a estimar tendría la siguiente función de verosimilitud en la RBNT tipo NB2: 
En donde:

》 es la matriz de variables independientes y de los parámetros beta a estimar.

" es el parámetro auxiliar de dispersión en la función binomial negativa que permite considerar la sobredispersión.

» es el valor de la distribución Gamma (la naturaleza de esta distribución se puede consultar en Hilbe (2014).

Para analizar si el modelo truncado NB2 consideró significativamente la sobredispersión, se realizó el Test de la Razón de Verosimilitud para estimar la significancia estadística del parámetro de dispersión a (alfa), bajo la hipótesis nula de $\alpha=0$. El "no rechazo de la hipótesis nula", reduciría el modelo a una Regresión Poisson Truncada de acuerdo a lo recomendado por Hilbe (2014) y Scott y Freese (2014), por lo que el modelo RBNT no sería adecuado para esta investigación.

\section{Modelo de fatalidad peatonal}

Para el caso de la estimación del Modelo de FP (hubo al menos un peatón muerto hubo sólo uno o más peatones heridos), se optó por una Regresión Logística Binaria. De acuerdo a Fávero y Belfiore (2019); Hoffman (2019) y Yang (2019), la ecuación general para la Regresión Logística Binaria estimada es del tipo:

Y cuya parametrización en $\mathrm{z}$ es:

\section{4}

Para este modelo la bondad de ajuste se estimó y analizó a través de la Prueba de Hosmer-Lemeshow bajo la hipótesis nula de que el modelo se ajusta a lo observado de acuerdo a lo recomendado por Hosmer et al. (2013). Igualmente se estimó el , para cada variable independiente, los cuales son una medida de la influencia explicativa de dicha variable sobre la respuesta. Los odds ratio menores a uno, se interpretan estimando su inversa 1 /odds ratio.

Finalmente, para seleccionar las variables que permanecieron en los modelos estimados, se consideró el Criterio de Información de Aikake (AIC) y la significancia de la Prueba de Wald. En el caso de AIC: se prefiere de un conjunto de modelos estimados, aquel que tenga el menor valor del Criterio estimado. Lo anterior permitió obtener los mejores modelos que explicaron el comportamiento de las variables dependientes.

Igualmente, debido a la naturaleza estimativa de los modelos, se analizaron posibles problemas de multicolinealidad mediante los Índices de Condición (uno por cada parámetro estimado) y el Criterio de Proporción de Descomposición de la Varianza (CPDV) por variable. Considerando que pudiese existir colinealidad de moderada a fuerte con índices entre 10 a 30, o muy fuerte por arriba de 30, siempre y cuando haya al menos un par de variables con CPDV $\geq 0.5$ en los Índices de Condición estimados (Ramírez y Ramírez, 2006).

La estimación de los modelos y pruebas estadísticas se realizó en el Programa Stata versión 15, siguiendo los procedimientos sugeridos por Greene (2008), Hilbe (2011, 2014), Cameron y Trivedi (2013), Hosmer et al. (2013) y Scott y Freese (2014). 


\section{Resultados y análisis de resultados}

\section{Frecuencia de Colisiones y mortalidad}

La frecuencia de CP ocurridas en el intervalo de años 2004 a 2015 (Cuadro 5) permite observar que, en promedio, el número de $\mathrm{CP}$ alcanza su pico más alto en viernes y desciende el sábado y domingo. El promedio sube el lunes y vuelve a descender hasta el miércoles. Los resultados anteriores coinciden con lo encontrado por Zhou et al. (2016) quienes dirigen su atención a los fines de semana (viernes y sábado) como días pico en cuanto al número de accidentes peatonales en zonas urbanas. Es por lo que autores como Baker et al. (1974); Quistberg et al. (2014) y Shinar (2017) mencionan la necesidad de analizar los posibles factores sociales implicados. Entre esos factores se podría tener el consumo de alcohol (tanto en conductores como en peatones) durante el inicio del fin de semana y días de asueto. Tomando en cuenta también, el posible consumo de alcohol de conductores y peatones en horario nocturno.

Respecto a la variable horario, investigadores como Zhou et al. (2016) y Shinar (2017) señalan que el horario influye en la frecuencia de accidentes peatonales considerando que durante horas de oscuridad puede ser menor la visibilidad y claridad para caminar y conducir en los espacios públicos. Determinar esta relación podría contribuir a implementar o fortalecer estrategias preventivas relacionadas con controles de consumo de alcohol al manejar. $\mathrm{O}$ bien contribuir a mejorar la visibilidad en vías públicas a través del alumbrado público o implementar medidas que permitan mejorar la visibilidad en los cruces peatonales.

Cuadro 5. Número de CP (frecuencia) por día de la semana ocurridos en zonas urbanas de CDMX (años 2004 a 2015). Fuente: elaboración personal con datos de INEGI (2020).

\begin{tabular}{|c|c|c|c|c|c|c|c|}
\hline \multirow{2}{*}{ Año } & \multicolumn{7}{|c|}{ Día de la semana } \\
\cline { 2 - 8 } & Lunes & Martes & Miércoles & Jueves & Viernes & Sábado & Domingo \\
\hline 2004 & 177 & 170 & 152 & 136 & 149 & 148 & 117 \\
\hline 2005 & 152 & 137 & 110 & 127 & 152 & 156 & 114 \\
\hline 2006 & 163 & 138 & 157 & 151 & 169 & 158 & 115 \\
\hline 2007 & 156 & 133 & 152 & 153 & 148 & 167 & 106 \\
\hline 2008 & 161 & 141 & 151 & 123 & 166 & 159 & 109 \\
\hline 2009 & 124 & 146 & 165 & 147 & 158 & 171 & 116 \\
\hline 2010 & 179 & 144 & 151 & 163 & 176 & 153 & 125 \\
\hline 2011 & 155 & 139 & 153 & 136 & 168 & 152 & 127 \\
\hline 2012 & 184 & 191 & 171 & 187 & 195 & 176 & 144 \\
\hline 2013 & 166 & 153 & 141 & 158 & 199 & 170 & 119 \\
\hline 2014 & 144 & 160 & 130 & 160 & 167 & 130 & 119 \\
\hline 2015 & 108 & 114 & 104 & 117 & 108 & 86 & 102 \\
\hline Total & 1.869 & 1.766 & 1.737 & 1.758 & 1.955 & 1.826 & 1.413 \\
\hline Media & 155,75 & 147,17 & 144,75 & 146,50 & 162,92 & 152,17 & 117,75 \\
\hline
\end{tabular}

En el Cuadro 6 se observa que en promedio el número de $\mathrm{CP}$ alcanza su cúspide en la tarde y desciende en la noche. Este comportamiento por horario, coincide con el encontrado en colisiones de vehículos con ciclistas (Issakson-Hellman, 2012; HernándezVásquez et al., 2016). Esto pudiera estar relacionado con el hecho de que la actividad urbana alcanza un pico durante horas de la tarde por lo que las $\mathrm{CP}$ alcanzan su pico en 
dicho horario. Empero, en las noches, aunque la actividad peatonal desciende, las CP son mayores a las ocurridas en los horarios restantes (madrugada y mañana).

Tomando en cuenta la ley de la capacidad cognitiva de la teoría de la causalidad hay que considerar que factores como el horario (asociado a condiciones de oscuridad/ visibilidad) y el consumo de alcohol por parte de los actores, pudiesen limitar la capacidad cognitiva de las personas, al afectar sus habilidades.

Cuadro 6. No. de colisiones con peatones (2004-2015) en zonas urbanas por horario (madrugada, mañana, tarde y noche). Fuente: elaboración personal con datos de INEGI (2020).

\begin{tabular}{|l|c|c|c|c|}
\hline \multirow{2}{*}{ Horario } & \multicolumn{4}{|c|}{ Horario del día } \\
\cline { 2 - 5 } & Madrugada & Mañana & Tarde & Noche \\
\hline 2004 & 67 & 267 & 418 & 297 \\
\hline 2005 & 89 & 231 & 325 & 303 \\
\hline 2006 & 78 & 273 & 390 & 310 \\
\hline 2007 & 92 & 269 & 371 & 283 \\
\hline 2008 & 93 & 243 & 388 & 286 \\
\hline 2009 & 112 & 253 & 374 & 288 \\
\hline 2010 & 122 & 269 & 375 & 325 \\
\hline 2011 & 143 & 247 & 343 & 297 \\
\hline 2012 & 148 & 348 & 381 & 371 \\
\hline 2013 & 146 & 282 & 365 & 313 \\
\hline 2014 & 94 & 267 & 312 & 337 \\
\hline 2015 & 82 & 220 & 239 & 198 \\
\hline Total & 1.266 & 3.169 & 4.281 & 3.608 \\
\hline Media & 106 & 264 & 357 & 301 \\
\hline
\end{tabular}

En cuanto al lugar de accidente (Cuadro 7), las CP en términos promedio son mayores en intersecciones que en las no intersecciones. En este análisis es necesario considerar que los lugares de cruce favorecidos técnicamente por la planeación urbana son las intersecciones, las cuales tendrían que contar con semáforos y otras señalizaciones. Esta motivación fomenta que la frecuencia de cruce peatonal de vialidades sea mayor en intersecciones que en no intersecciones. Empero hay que cuestionar si ¿ ¿se debe esperar una mayor frecuencia y promedio de colisiones en intersecciones? Por asociación, se podría esperar una mayor cantidad de colisiones en intersecciones.

Ahora bien, fomentar atravesar las vías en intersecciones, afectaría directamente la ley natural del aprendizaje de la teoría de causalidad, ya que propiciaría el uso continuo e intensivo de las intersecciones como lugares de cruce peatonal. Empero no todos los peatones atraviesan en las esquinas de las calles, avenidas o andadores y no todas las esquinas cuentan con semáforos y señalizaciones. Por lo anterior serán, necesarios futuros estudios, que determinen frecuencias de cruce peatonal en intersección y no intersección, junto con estudios de cantidad/calidad de semáforos y señalizaciones en vialidades para explicar la mayor frecuencia de colisiones en intersecciones. Sin embargo, ¿qué impacto en la mortalidad tendrá el cruce en intersección y en no intersección? Esto se podrá contestar más adelante con el modelo de fatalidad peatonal.

El aspecto anterior, necesita ser abordado también a futuro, considerando puntualmente el comportamiento para atravesar vialidades de los diversos tipos de peatón, junto con las motivaciones que implican la toma de decisiones de atravesar en una intersección o en no intersección. Lo anterior se ha relacionado con el hecho de que los peatones no 
caminan en línea recta, sino que se pueden desplazar irregularmente y cruzar las vías en los puntos de no intersección para ahorrar tiempo o por otro motivo (Jiménez, 2010; Espinosa, 2013; Papadimitriou et al., 2017; Aristizábal et al., 2018). Posteriores investigaciones, podrían considerar el enfoque de la psicología y de la economía conductual; por ejemplo, a través de la teoría de la prospectiva, (aversión/adicción al riesgo) aplicada a la decisión del peatón por cruzar o no en intersecciones (Shaoman y Ying, 2015).

Cuadro 7. Número de CP por lugar de accidente en zonas urbanas de CDMX (2004-2015). Fuente: elaboración personal con datos de INEGI (2020).

\begin{tabular}{|l|c|c|}
\hline \multirow{2}{*}{ Año } & \multicolumn{2}{|c|}{ Lugar del accidente } \\
\cline { 2 - 3 } & Accidente en intersección & Accidente en no intersección \\
\hline 2004 & 751 & 298 \\
\hline 2005 & 611 & 337 \\
\hline 2006 & 686 & 365 \\
\hline 2008 & 631 & 384 \\
\hline 2009 & 669 & 341 \\
\hline 2010 & 699 & 328 \\
\hline 2011 & 736 & 355 \\
\hline 2012 & 614 & 416 \\
\hline 2013 & 779 & 469 \\
\hline 2014 & 725 & 381 \\
\hline 2015 & 625 & 385 \\
\hline Total & 503 & 236 \\
\hline Promedio & 8.029 & 4.295 \\
\hline
\end{tabular}

Cuadro 8. Número de peatones heridos por CP en cada día de la semana en zonas urbanas de CDMX (2004-2015). Fuente: elaboración personal con datos de INEGI (2020).

\begin{tabular}{|l|c|c|c|c|c|c|c|}
\hline \multirow{2}{*}{ Año } & \multicolumn{7}{|c|}{ Día de la semana } \\
\cline { 2 - 8 } & Lunes & Martes & Miércoles & Jueves & Viernes & Sábado & Domingo \\
\hline 2004 & 170 & 162 & 153 & 130 & 131 & 141 & 106 \\
\hline 2005 & 140 & 134 & 99 & 120 & 141 & 149 & 93 \\
\hline 2006 & 145 & 126 & 150 & 137 & 144 & 145 & 103 \\
\hline 2007 & 157 & 115 & 140 & 150 & 141 & 139 & 95 \\
\hline 2008 & 156 & 130 & 134 & 117 & 161 & 146 & 96 \\
\hline 2009 & 122 & 139 & 166 & 137 & 155 & 158 & 107 \\
\hline 2010 & 167 & 133 & 130 & 153 & 171 & 155 & 116 \\
\hline 2011 & 146 & 130 & 144 & 119 & 158 & 142 & 109 \\
\hline 2012 & 168 & 178 & 157 & 179 & 189 & 159 & 129 \\
\hline 2013 & 153 & 151 & 127 & 157 & 190 & 153 & 101 \\
\hline 2014 & 128 & 148 & 116 & 150 & 154 & 127 & 118 \\
\hline 2015 & 101 & 111 & 106 & 114 & 102 & 77 & 97 \\
\hline Total & 1.753 & 1.657 & 1.622 & 1.663 & 1.837 & 1.691 & 1.270 \\
\hline Media & 146,08 & 138,08 & 135,17 & 138,58 & 153,08 & 140,92 & 105,83 \\
\hline
\end{tabular}

En cuanto a peatones heridos, su número promedio en $\mathrm{CP}$ es mayor el viernes con respecto al resto de días de la semana: desciende hacía el sábado y domingo. No obstante, el promedio del sábado es inferior al del lunes, día a partir del cual hay un descenso hasta el miércoles (Cuadro 8.). 
Sobre promedio de peatones muertos: la mayor mortalidad se presenta los días sábado, descendiendo a partir del domingo hasta el martes. (Cuadro 9).

Cuadro 9. Número de peatones muertos en CP en cada día de la semana en zonas urbanas de CDMX (2004-2015). Fuente: elaboración personal con datos de INEGI (2020).

\begin{tabular}{|l|c|c|c|c|c|c|c|}
\hline \multirow{2}{*}{ Día } & \multicolumn{7}{|c|}{ Día de la semana } \\
\cline { 2 - 9 } & Lunes & Martes & Miércoles & Jueves & Viernes & Sábado & Domingo \\
\hline 2004 & 17 & 17 & 6 & 14 & 26 & 21 & 14 \\
\hline 2005 & 20 & 15 & 15 & 14 & 22 & 23 & 28 \\
\hline 2006 & 24 & 20 & 18 & 22 & 32 & 28 & 21 \\
\hline 2007 & 14 & 28 & 23 & 18 & 20 & 39 & 19 \\
\hline 2008 & 15 & 17 & 24 & 9 & 19 & 22 & 21 \\
\hline 2009 & 13 & 17 & 17 & 18 & 19 & 29 & 20 \\
\hline 2010 & 26 & 19 & 27 & 24 & 22 & 19 & 27 \\
\hline 2011 & 17 & 17 & 25 & 22 & 20 & 20 & 26 \\
\hline 2012 & 29 & 20 & 23 & 24 & 21 & 30 & 24 \\
\hline 2013 & 28 & 19 & 22 & 17 & 31 & 29 & 30 \\
\hline 2014 & 24 & 19 & 24 & 23 & 23 & 22 & 15 \\
\hline 2015 & 15 & 11 & 8 & 13 & 16 & 14 & 15 \\
\hline Total & 242 & 219 & 232 & 218 & 271 & 296 & 260 \\
\hline Media & 20,17 & 18,25 & 19,33 & 18,17 & 22,58 & 24,67 & 21,67 \\
\hline
\end{tabular}

Sobre mortalidad peatonal considerando horarios de madrugada, mañana, tarde y noche (Cuadro 10), hay que decir que el número promedio de heridos alcanza el mayor valor en las tardes descendiendo hasta la madrugada. Mientras que la frecuencia de peatones muertos es mayor durante la noche con respecto a las tardes descendiendo en la madrugada y volviéndose a incrementar en las mañanas. Nuevamente recordando la ley de la capacidad cognitiva de la teoría, se tiene que considerar la posibilidad de que el horario (asociado a condiciones de visibilidad/oscuridad) pudiera imponer límites al procesamiento de la información de los conductores/peatones. Las limitaciones podrían resultar en una mayor mortalidad nocturna. Futuras investigaciones en CDMX deberán abordar la mortalidad y fatalidad de los accidentes considerando condiciones de particulares de visibilidad y hora del accidente.

Cuadro 10. Número de peatones heridos y muertos en CP en horas de madrugada, mañana, tarde y noche en zonas urbanas de CDMX (2004-2005). Fuente: elaboración personal con datos de INEGI (2020).

\begin{tabular}{|l|c|c|c|c|c|c|c|c|}
\hline \multirow{2}{*}{ Año } & \multicolumn{4}{|c}{ Número de peatones heridos } & \multicolumn{4}{c|}{ Número de peatones muertos } \\
\cline { 2 - 10 } & Madrugada & Mañana & Tarde & Noche & Madrugada & Mañana & Tarde & Noche \\
\hline 2004 & 55 & 246 & 401 & 291 & 14 & 30 & 43 & 28 \\
\hline 2005 & 78 & 209 & 317 & 272 & 21 & 34 & 26 & 56 \\
\hline 2006 & 61 & 242 & 372 & 275 & 22 & 41 & 47 & 55 \\
\hline 2007 & 66 & 237 & 367 & 267 & 30 & 50 & 36 & 45 \\
\hline 2008 & 69 & 233 & 374 & 264 & 28 & 21 & 39 & 39 \\
\hline 2009 & 95 & 242 & 379 & 268 & 24 & 31 & 32 & 46 \\
\hline 2010 & 115 & 240 & 369 & 301 & 21 & 46 & 40 & 57 \\
\hline 2011 & 120 & 218 & 329 & 281 & 33 & 40 & 32 & 42 \\
\hline 2012 & 126 & 310 & 381 & 342 & 31 & 56 & 31 & 53 \\
\hline 2013 & 106 & 262 & 359 & 305 & 46 & 43 & 39 & 48 \\
\hline
\end{tabular}




\begin{tabular}{|l|c|c|c|c|c|c|c|c|}
\hline 2014 & 74 & 242 & 307 & 318 & 24 & 41 & 33 & 52 \\
\hline 2015 & 71 & 219 & 227 & 191 & 19 & 26 & 28 & 19 \\
\hline Total & 1.036 & 2.900 & 4.182 & 3.375 & 313 & 459 & 426 & 540 \\
\hline Media & 86,33 & 241,67 & 348,5 & 281,25 & 26,08 & 38,25 & 35,5 & 45,0 \\
\hline
\end{tabular}

Finalmente, en cuanto al lugar de la colisión, tanto el promedio de peatones heridos y muertos es mayor en intersecciones que en no intersecciones, tendiendo a ser mayor el número promedio de peatones heridos que el número promedio de peatones muertos (Cuadro 11).

Cuadro 11. Número de peatones heridos y muertos en CP por lugar de accidente (intersección y no intersección de vialidades) en zonas urbanas de CDMX (2004 a 2015). Fuente: elaboración personal con datos de INEGI (2020).

\begin{tabular}{|l|c|c|c|c|}
\hline \multirow{2}{*}{ Año } & \multicolumn{2}{|c}{ Peatones heridos } & \multicolumn{2}{c|}{ Peatones muertos } \\
\cline { 2 - 5 } & $\begin{array}{c}\text { Accidente en } \\
\text { intersección }\end{array}$ & $\begin{array}{c}\text { Accidente en no } \\
\text { intersección }\end{array}$ & $\begin{array}{c}\text { Accidente en } \\
\text { intersección }\end{array}$ & $\begin{array}{c}\text { Accidente en no } \\
\text { intersección }\end{array}$ \\
\hline 2004 & 709 & 284 & 85 & 30 \\
\hline 2005 & 574 & 302 & 85 & 52 \\
\hline 2006 & 630 & 320 & 99 & 66 \\
\hline 2007 & 596 & 341 & 94 & 67 \\
\hline 2008 & 636 & 304 & 71 & 56 \\
\hline 2009 & 674 & 310 & 92 & 41 \\
\hline 2010 & 706 & 319 & 98 & 66 \\
\hline 2011 & 582 & 366 & 75 & 72 \\
\hline 2012 & 736 & 423 & 93 & 78 \\
\hline 2013 & 688 & 344 & 108 & 68 \\
\hline 2014 & 586 & 355 & 86 & 64 \\
\hline 2015 & 481 & 227 & 63 & 29 \\
\hline Total & 7.598 & 3.895 & 1.049 & 689 \\
\hline Promedio & 633,17 & 324,58 & 87,42 & 57,42 \\
\hline
\end{tabular}

\section{Modelos estimados}

Para evitar problemas de multicolinealidad en ambos modelos (CP y FP), para el caso de vehículos de transporte, se invirtió la categorización de la variable automóvil involucrado, quedando como "categoría base $0=$ sí" (Ver Cuadro 3). Siguiendo con el análisis de multicolinealidad, el modelo de CP tuvo 6 de 38 índices de condición, cuyos valores estuvieron entre 10,14 a un máximo de 24,03. El modelo de RP tuvo 6 de 41 índices que oscilaron entre 10,92 a un máximo de 26,29. Empero, en ambos casos no se obtuvieron pares de variables con CPDV mayores o iguales a 0,5 . Por lo que se concluye estadísticamente que no existen problemas de multicolinealidad que afecten las estimaciones de los parámetros en los modelos.

\section{Modelo estimado de colisiones}

Sobre los modelos estimados, en el modelo de CP (Cuadro 12.), se encontró que el resultado significativo del test de la razón de verosimilitud, permite concluir que el modelo truncado NB2 en esta investigación: considera la sobredispersión, por lo cual es adecuado para modelar las colisiones. 
El resultado de los parámetros de tiempo del modelo de colisiones (Cuadro 12.), permite sustentar la hipótesis de que es estadísticamente significativo el que los días viernes se incremente el número de colisiones con respecto a otros días entre semana, incluyendo sábado/domingo. Lo anterior es parcialmente acorde con las observaciones hechas por Hernández-Hernández y De Haro-De León (2014) sobre atropellamientos en Ciudad Juárez al norte de México, encontrando que los atropellamientos alcanzan su nivel más alto los viernes y sábados en ámbitos urbanos.

Además, si bien hay colisiones las 24 horas del día, el número de colisiones se incrementa significativamente en mañanas, noches y especialmente en las tardes con respecto a horas de madrugada. Este último resultado es acorde a lo encontrado por Shinar (2017) en ciudades de Estados Unidos. Al respecto, Pérez y Gómez (2014), mencionan que un alto porcentaje de muertes y lesiones por atropellamientos en el mundo, ocurre en condiciones que dificultan la visibilidad, como mala iluminación al atardecer y al anochecer. También, se observa un incremento significativo de $\mathrm{CP}$ en intersecciones con respecto a no intersecciones (Cuadro 12).

Con respecto a la variable "causa atribuible del accidente", en CDMX el número de colisiones peatonales es significativamente menor cuando la causa se atribuye al peatón y a otras causas que cuando se atribuye el conductor. Igualmente, la cantidad de colisiones se incrementa cuando hay un automóvil involucrado en la colisión. Siguiendo la teoría de causalidad y bajo la ley de capacidad cognitiva, la responsabilidad y procesamiento de información por parte del conductor es fundamental para evitar los atropellamientos. Si bien, son necesarios estudios particulares conductuales de la persona sobre decisiones que toman al cruzar la vía y manejar respectivamente, el conductor "particularmente de automóviles", podría ser un factor fundamental en la prevención de atropellamientos.

Cuadro 12. Estimación del Modelo de Colisiones (RBNT) y del Modelo de FP. Fuente: elaboración propia con datos provenientes de INEGI (2020). Nota 1: $*$ Nivel de significancia de al menos 0,01; **Nivel de significancia de al menos 0,05; "Nivel de significancia de al menos 0,1.

\begin{tabular}{|c|c|c|c|}
\hline \multicolumn{2}{|c|}{ Modelo de CP (RBNT). } & \multicolumn{2}{|c|}{ Modelo de FP (RL). } \\
\hline Variable. & $\begin{array}{c}\text { Parámetro } \\
\text { (error estándar robusto). }\end{array}$ & $\begin{array}{c}\text { Parámetro } \\
\text { (error estándar robusto). }\end{array}$ & Odds ratio. \\
\hline Constante. & $\begin{array}{c}-3,4740 * * * \\
(0,2264)\end{array}$ & $\begin{array}{c}-1,6770 * * * \\
(0,2411)\end{array}$ & 0,1869 \\
\hline Martes. & $\begin{array}{l}-0,0442 \\
(0,0660)\end{array}$ & $\begin{array}{l}0,0004 \\
(0,1049)\end{array}$ & 1,0004 \\
\hline Miércoles. & $\begin{array}{l}-0,0698 \\
(0,0670)\end{array}$ & $\begin{array}{c}0,0575 \\
(0,1029)\end{array}$ & 1,0592 \\
\hline Jueves. & $\begin{array}{l}-0,1186^{*} \\
(0,0651)\end{array}$ & $\begin{array}{l}-0,0193 \\
(0,1046)\end{array}$ & 0,9808 \\
\hline Viernes. & $\begin{array}{l}0,3626^{* * * *} \\
(0,1000)\end{array}$ & $\begin{array}{c}-0,4079^{* * * *} \\
(0,1413)\end{array}$ & 0,6650 \\
\hline Sábado. & $\begin{array}{l}-0,1218 * \\
(0,0713)\end{array}$ & $\begin{array}{l}0,2140 * * \\
(0,0978)\end{array}$ & 1,2386 \\
\hline Domingo. & $\begin{array}{c}-0,2844^{* * * *} \\
(0,0758)\end{array}$ & $\begin{array}{l}0.2952^{* * * *} \\
(0.1034)\end{array}$ & 1,3434 \\
\hline Mañana. & $\begin{array}{l}0,5199 * * * \\
(0,0817)\end{array}$ & $\begin{array}{l}-0,5105 * * * \\
(0,0889)\end{array}$ & 0,6002 \\
\hline Tarde. & $\begin{array}{c}0.6954^{* * *} \\
(0,0789)\end{array}$ & $\begin{array}{c}-1,0083^{* * *} \\
(0,0970)\end{array}$ & 0,3648 \\
\hline Noche. & $\begin{array}{l}0,5484 * * * \\
(0,0861)\end{array}$ & $\begin{array}{l}-0,5226 * * * \\
(0,0894)\end{array}$ & 0,5930 \\
\hline
\end{tabular}




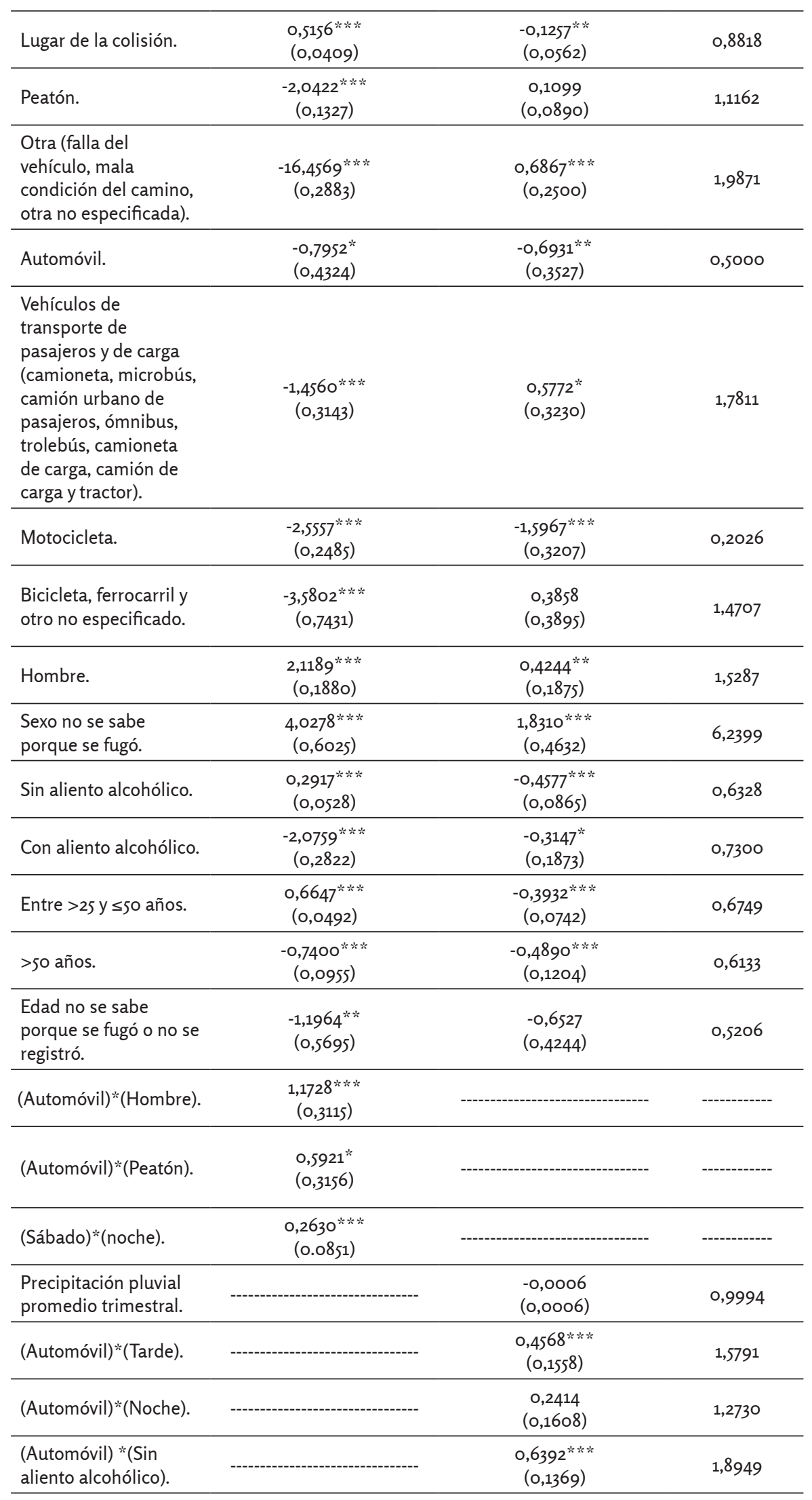




\begin{tabular}{|c|c|c|c|}
\hline $\begin{array}{l}\text { (Automóvil)* } \\
\text { (Precipitación pluvial } \\
\text { promedio trimestral). }\end{array}$ & -------------------- & $\begin{array}{l}0,0023^{*} \\
(0,0012)\end{array}$ & 1,0023 \\
\hline $\begin{array}{l}(\text { Motocicleta)*( } \\
\text { Hombre)*(Entre >25 y } \\
\leq 50 \text { años). }\end{array}$ & 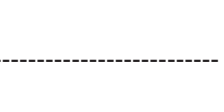 & $\begin{array}{c}1,0833^{* * *} \\
(0,3989)\end{array}$ & 2,9545 \\
\hline 2005 & $\begin{array}{l}-0,3190 * * * \\
(0,0918)\end{array}$ & $\begin{array}{l}0,3901^{* * * *} \\
(0,1408)\end{array}$ & 1,4772 \\
\hline 2006 & $\begin{array}{c}-0,1344 \\
(0,0893)\end{array}$ & $\begin{array}{l}0,5780 * * * \\
(0,1370)\end{array}$ & 1,7825 \\
\hline 2007 & $\begin{array}{l}-0,3330 * * * \\
(0,0899)\end{array}$ & $\begin{array}{c}0,4140^{* * *} \\
(0,1431)\end{array}$ & 1,5129 \\
\hline 2008 & $\begin{array}{l}-0,1376 \\
(0,0894)\end{array}$ & $\begin{array}{l}0,2753^{*} \\
(0,1501)\end{array}$ & 1,3169 \\
\hline 2009 & $\begin{array}{c}-0,1414 \\
(0,0926)\end{array}$ & $\begin{array}{c}0,1964 \\
(0,1506)\end{array}$ & 1,2171 \\
\hline 2010 & $\begin{array}{l}-0,1591^{*} \\
(0,0887)\end{array}$ & $\begin{array}{l}0,2879^{* *} \\
(0,1456)\end{array}$ & 1,3337 \\
\hline 2011 & $\begin{array}{l}-0,2643^{* * * *} \\
(0,0900)\end{array}$ & $\begin{array}{l}-0,0013 \\
(0,1485)\end{array}$ & 0,9987 \\
\hline 2012 & $\begin{array}{l}-0,0887 \\
(0,0836)\end{array}$ & $\begin{array}{c}0,0473 \\
(0,1448)\end{array}$ & 1,0484 \\
\hline 2013 & $\begin{array}{l}-0,4818 * * * \\
(0,0930)\end{array}$ & $\begin{array}{c}0,2031 \\
(0,1408)\end{array}$ & 1,2252 \\
\hline 2014 & $\begin{array}{l}-0,4580 * * * \\
(0,0978)\end{array}$ & $\begin{array}{c}0,1335 \\
(0,1469)\end{array}$ & 1,1428 \\
\hline 2015 & $\begin{array}{c}-0,6984^{* * *} \\
(0,1212)\end{array}$ & $\begin{array}{l}-0,0549 \\
(0,1653)\end{array}$ & 0,9465 \\
\hline Ln Alfa. & $\begin{array}{c}-1,7274 \\
(0,2470)\end{array}$ & ---------------------- & ---------- \\
\hline Alfa. & $\begin{array}{c}0,1778 \\
(0,0439)\end{array}$ & -------------------- & ---------- \\
\hline \multicolumn{2}{|l|}{$\mathrm{n}=9.218$ observaciones. } & \multicolumn{2}{|c|}{$\mathrm{n}=12.324$} \\
\hline \multicolumn{2}{|c|}{ Test de Wald $[\mathrm{Ji} 2(37 \mathrm{gl})]=4.695,90^{* * *}$} & \multicolumn{2}{|c|}{ Test de Wald $[\mathrm{ji} 2(40)]=734,73^{* * *}$} \\
\hline \multicolumn{2}{|c|}{ Log pseudolikelihood $=-5.855,8221 ; \mathrm{AIC}=11.789,64$} & \multicolumn{2}{|c|}{$\begin{array}{c}\text { Log pseudolikelihood= }-4.523,8313 ; \\
\text { AIC }=9.129,663\end{array}$} \\
\hline \multicolumn{2}{|c|}{$\begin{array}{l}\text { Test de la razón de verosimilitud para Ho: Alfa }=0 \\
\text { LR chi2(1) }=28,06^{* * *}\end{array}$} & \multicolumn{2}{|c|}{ Test de Hosmer-Lemeshow $\operatorname{chi} 2(8)=14,12^{*}$} \\
\hline
\end{tabular}

Los resultados sobre sexo del conductor (Cuadro 12) coinciden con resultados de Elvik (2006) y Quistberg et al. (2014) en Noruega y Perú respectivamente, en cuanto a que las colisiones se incrementan cuando el conductor es hombre con respecto a si es mujer (categoría base del estudio). No obstante, el número de colisiones tiende a incrementarse más en el caso de conductores fugados (de los que se desconoce el sexo). Por lo anterior, los resultados encontrados deben tomarse con reserva, ya que de acuerdo a INEGI (2020), las fugas además de evitar acciones legales, impiden conocer sexo, edad y posible alcoholismo de quien manejó el vehículo.

Sobre aliento alcohólico, se debe interpretar también con reserva la significancia estadística de la variable "con aliento alcohólico del conductor", la cual disminuye el número de colisiones con respecto a la categoría base "no se sabe porque se fugó o no se registró". Como señala Tabasso (2011), aparte del olor a aliento alcohólico, no hay un sólo síntoma que debido al consumo del alcohol no pueda ser encontrado también en algún otro estado patológico. Además, la constatación aislada del aliento alcohólico es muy desconfiable en investigaciones rigurosas. 
Por lo anterior, sería más confiable una medición del grado de intoxicación alcohólica en conductor y peatón, y así analizar el influjo del alcohol en las CP. De acuerdo a Shinar (2017) es necesario evaluar la presencia de alcohol en sangre de peatones atropellados. Por ejemplo, en Suecia, Öström y Eriksson (2001), encontraron alcohol en un 24\% de peatones masculinos muertos por atropellamiento. Lo anterior es importante para el deslinde de responsabilidades del accidente y también para elaborar estrategias de prevención dirigidas al conductor y al peatón. Empero, las fugas del conductor, son un problema que impide la acción de la justicia, además de impedir conocer aspectos sociodemográficos del conductor, aspectos que de conocerse, permitirían desarrollar estrategias de intervención para evitar accidentes de acuerdo al modelo teórico de seguridad vial.

En las categorías de edad del conductor (Cuadro 12), el número de colisiones se incrementa en un rango de edad de mayor a 25 hasta 50 años con respecto a la categoría base ( $\leq 25$ años) y disminuye en conductores mayores de 50 años y de conductores de los cuales se desconoce la edad. Igualmente estos resultados deben tomarse con reserva por la fuga de los conductores. Al respecto Elvik (2016), menciona que los conductores completan su aprendizaje a los 25 años, por lo que se esperaría una frecuencia estable de accidentes a partir de los 25 años en adelante. Empero Elvik, también señala que el conducir largas distancias anuales aparentemente está asociado con más éxito en evitar accidentes, independientemente de la edad del conductor. Futuras investigaciones deberán considerar esta posibilidad.

En este modelo de colisiones (Cuadro 12), resultaron positivamente significativas las interacciones (Automóvil)*(Hombre) y (Automóvil)*(Peatón). En ambos casos se debe interpretar considerando la categoría base de la variable Automóvil. Por lo que las interacciones indican que cuando el conductor es hombre y cuando la causa es atribuible al peatón, se tiende a incrementar el número de colisiones cuando no hay automóviles involucrados. En el caso de la interacción (Sábado) ${ }^{\star}($ Noche), también se encontró, un incremento estadísticamente significativo en el número de colisiones.

En cuanto a los años de análisis se observa una tendencia a disminuir el número de CP con respecto al año 2004, para los años 2005, 2007, 2010, 2011, 2013, 2014 y 2015 (Cuadro 12).

Finalmente, sobre las variables de lluvia y temperatura y sus respectivas interacciones con otras variables independientes: no fueron significativas, ni aportaban al modelo final de acuerdo al Criterio de Información de Aikake, por lo que se decidió dejarlas fuera del modelo final estimado, permitiendo así su parsimonia. Sobre variables de clima, sería recomendable que futuros estudios en CDMX analicen de manera particular si existe alguna relación causal y/o asociación con la frecuencia de CP y su mortalidad.

\section{Modelo estimado de fatalidad}

En primer lugar, sobre el Test de Hosmer-Lemeshow, hay que señalar que bajo la hipótesis nula de que el modelo se ajusta a los datos observados, dicha hipótesis no se rechaza considerando niveles de significancia de 0,05 y 0,01 . Por lo que se puede concluir bajo esos niveles que el modelo presenta bondad de ajuste.

Considerando los signos de los parámetros y los odds ratio estimados en este modelo (Cuadro 12), se encontró que la probabilidad de una fatalidad es menor en mañana, tarde y noche que en madrugada. Se tiene que tomar en cuenta que si bien el número de colisiones aumenta en la noche y especialmente en tarde con respecto a madrugada; la probabilidad de la fatalidad tiene un comportamiento inverso. Considerando el 
"sistema de responsabilidad de tres fases", cabe cuestionar el por qué se presenta una mayor fatalidad en ese horario. (Wegman, 2001; Bliss, 2004). Para contestar lo anterior, se sugiere evaluar en futuros estudios, los tiempos y calidad de atención médica de emergencia que se proporciona a los atropellados en diversas horas.

Por otra parte, observando el signo de los parámetros de las variables días de la semana, se advierte que la fatalidad es más probable en sábado. Sin embargo, se observó con anterioridad que el número de colisiones peatonales se incrementa en viernes. Incluso para fines de semana, los odds ratio estimados permiten señalar que los sábados y especialmente los domingos son los que más explican la probabilidad de FP (Cuadro 12).

Con respecto al lugar del accidente, los signos de los parámetros estimados (Cuadro 12), indica que la probabilidad de fatalidad disminuye en intersecciones con respecto a las no intersecciones. Hay que considerar en este análisis que si bien el número de colisiones es mayor en intersecciones, empero la FP tiende a ser más probable en no intersecciones (Cuadro 12). Esto evidencia el riesgo mortal del comportamiento del peatón al atravesar en no intersección. Este hecho pudiera explicarse mediante la ley de eventos raros, considerando que siendo las intersecciones lugares más adecuados para atravesar, el cruce en no intersección al ser un evento raro con respecto al anterior incrementa su efecto en la tasa de accidentes. Resultando en una colisión con mayor probabilidad de fatalidad.

Por lo anterior, es necesario que investigaciones posteriores analicen a detalle la conducta y decisiones del peatón para elegir cruzar las vialidades en intersección o en no intersección. Además de que se podría evaluar a más detalle la probabilidad considerando si los peatones son o no adictos al riesgo en términos de la teoría de la prospectiva de Kahneman y Tversky (Shaoman y Ying, 2015; Chen, 2016).

Por otra parte, los signos obtenidos para las variables de vehículos de transporte (Cuadro 12), indican que la probabilidad de FP disminuye si no hay automóvil involucrado en la CP con respecto a sí hay automóviles involucrados. En el caso de vehículos de transporte de pasajeros y de carga involucrados, su presencia incrementa la probabilidad de la fatalidad. Empero de estas dos variables, son los automóviles los que más explican dicha probabilidad (ver odds ratio). En el caso de motocicletas involucradas, su presencia tiende a disminuir la probabilidad de la fatalidad, caso contrario al de bicicletas y otro tipo de vehículos involucrados.

Considerando los signos que acompañan a los parámetros de la variable sexo (Cuadro 12), se tiene que la probabilidad de la fatalidad se incrementa en el caso de conductores de los que se desconoce sexo porque se fugaron y también de hombres con respecto a mujeres conductoras. Continuando con análisis de signos: se puede leer que la probabilidad de la FP disminuye cuando el conductor tenga o no tenga aliento alcohólico, con respecto a que se desconozca su aliento porque se fugó o no se registró. El signo negativo de los parámetros de edad, incluyendo cuando la edad no se sabe porque se fugó o no registro, indica que la probabilidad de la fatalidad disminuye con respecto a si el conductor tiene 25 o menos años. Se insiste que estos resultados deben tomarse con reserva debido a la fuga de los conductores.

En el modelo de FP estimado, se observa una significancia estadística de cuatro variables de interacción (Automóvil)*(Tarde); (Automóvil)*(Sin aliento alcohólico); (Automóvil $)^{\star}($ Precipitación pluvial promedio trimestral) además de la interacción

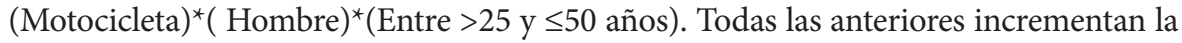
probabilidad de que la colisión sea fatal. A recordar que la variable automóvil se interpreta considerando: $0=$ sí hay automóvil involucrado, $1=$ no lo hay. Se observa que la variable "precipitación pluvial promedio trimestral" per se, no es significativa en el modelo. 
La variable temperatura y su interacción con el resto de las variables independientes (incluyendo precipitación), no fueron significativas dentro del modelo. Por lo que de acuerdo con el Criterio de Información de Aikake, se decidieron dejar fuera del modelo final estimado para mantener una parsimonia. Empero investigaciones futuras deberían seguir analizando posibles efectos del clima en la fatalidad peatonal.

Con respecto a fatalidad anual, se observa un incremento de probabilidad de FP para los años 2005, 2006, 2007, 2008, 2009, 2010, 2012, 2013, 2014 considerando el año base 2004, caso contario para los años 2011 y 2015. Los resultados de FP anual, deben considerarse con reserva, ya que Pérez y Gómez (2014), citan una tendencia nacional por subestimar mortalidad. Por ejemplo, ellos citan que un número importante de certificados de defunción carecen de información que impide registrar adecuadamente la mortalidad por atropellamiento.

Considerando los resultados y análisis de esta investigación es necesario tomar en cuenta, que este trabajo está acotado por diversas circunstancias que se han comentado a lo largo del texto, de ninguna manera pretende sustituir en importancia ni rigor a otras investigaciones empíricas de CP y FP. En el entendido que muchas de ellas van dirigidas a mejorar los procesos de seguridad vial para prevenir lesiones por atropellamientos tal como lo describen Rodríguez et al. (2014).

De esta forma, los resultados de esta investigación pretenden con todas sus limitantes ser complementarias a estudios e investigaciones de índole geográfica, de ingeniería del transporte y de salud pública. Los modelos estimados y analizados en este trabajo, como se señaló anteriormente, son de tipo estimativo más no predictivo, a diferencia de otros estudios de colisiones vehiculares, de corte más predictivo que estimativo como el realizado por Pérez y Gómez (2014). Finalmente, es necesario que futuras investigaciones incorporen a los diversos tipos de peatón según su grado de vulnerabilidad ante las colisiones con vehículos y sus interacciones en el espacio público.

Igualmente, es necesario que nuevos estudios incorporen al ciclista y al motociclista como sujetos vulnerables, aunque es un hecho, como se mencionó anteriormente, que el peatón es de todos ellos el más vulnerable. También se deben considerar a futuro, las aportaciones que puedan hacer la psicología, la economía conductual, la geografía y la planeación urbana en el análisis temporal y espacial de las colisiones vehiculares. Es necesario que esos estudios puedan tomar en cuenta, aspectos particulares de la conducta, además de las señalizaciones de tránsito vehicular, la velocidad vehicular y las condiciones físicas/ ambientales presentes en las colisiones peatonales, tal como lo describe Helmer (2015).

\section{Conclusiones}

Para el horizonte de tiempo analizado (2004 a 2015), se concluye y destacan los siguientes aspectos en cuanto al comportamiento de las colisiones peatonales y su fatalidad de en zonas urbanas de CDMX.

En cuanto a colisiones, en términos promedio la frecuencia de atropellamientos alcanzan sus valores más altos los viernes. Empero, en términos promedio la mortalidad es mayor en sábados. Siendo siempre mayor el número de heridos al número de muertos todos los días. En cuanto a horario, las CP se incrementan en la tarde y desciende en la noche. Además, el conteo nocturno es mayor al conteo de madrugada y mañana. Por otra parte, en promedio el mayor número de heridos se alcanza en la tarde descendiendo hasta la madrugada. Sin embargo, el promedio de peatones muertos es mayor en 
noche con respecto a tarde. Con respecto a lugar, se encontró que el número promedio de colisiones, de peatones muertos y de peatones heridos es mayor en intersecciones que en no intersecciones, siendo mayor el promedio de heridos al de muertos.

Los resultados del modelo de CP, permiten concluir que en CDMX durante el período 2004 a 2015, los viernes se incrementa significativamente el conteo de colisiones con respecto a otros días de la semana. Igualmente, el número de colisiones aumenta especialmente en tardes con respecto a madrugada y cuando hay un automóvil involucrado.

$\mathrm{El}$ incremento de CP también es estadísticamente significativo en intersecciones. Además, se encontró que el número de colisiones disminuye cuando la causa se atribuye al peatón u otras causas diferentes al conductor. Esto último pudiera indicar que la responsabilidad y procesamiento de información por parte del conductor, es fundamental para evitar los atropellamientos según la ley de capacidad cognitiva de la teoría de causalidad.

Se encontró también que el número de colisiones se incrementa significativamente en el caso de conductores que se fugan y de los que se desconoce el sexo. Por lo mismo, se debe interpretar con reserva la significancia estadística de la variable "con aliento alcohólico del conductor", la cual disminuye el número de colisiones con respecto a la categoría base en la cual se desconoce si el conductor tenía aliento alcohólico porque se fugó o no se registró. Respecto a edad del conductor, el número de colisiones se incrementa en un rango de edad de 25 a 50 años y disminuye en conductores mayores de 50 años y de conductores de los cuales se desconoce la edad.

Los resultados del modelo de FP permiten concluir que si bien las colisiones tienden a incrementarse en la tarde con respecto a noche y madrugada; en el caso de la fatalidad, su probabilidad es menor en las tardes y noches con respecto a madrugadas. Por esto, siguiendo la fase de intervención del modelo teórico de manejo de seguridad, se hace necesario evaluar en futuras investigaciones, los tiempos de atención médica a los atropellados en diferentes horarios.

Aunque el número de colisiones se incrementa significativamente en viernes, la fatalidad es más probable en sábado y domingo. Incluso los odds ratio estimados, señalan que los sábados y especialmente los domingos son los que más explican la probabilidad de FP. Sobre lugar del accidente, la probabilidad de fatalidad disminuye en intersecciones con respecto a no intersecciones. Empero, aunque el número de colisiones es mayor en intersecciones: la fatalidad tiende a ser más probable en no intersecciones. Este resultado evidencia el riesgo mortal que tiene el peatón al atravesar en no intersección, por lo cual es necesario que futuros estudios analicen a más detalle la conducta del peatón en los lugares de cruce considerando teorías psicológicas y/o de economía conductual.

Se concluye que la probabilidad de una fatalidad disminuye si no hay automóvil involucrado en la colisión con respecto a sí hay automóviles involucrados. También se encontró, que la probabilidad de fatalidad se incrementa cuando los conductores son hombres o se fugan que cuando son mujeres conductoras. El involucramiento por sexo del conductor coincide con resultados encontrados en otros estudios. Sin embargo, los resultados por sexo deben tomarse con reserva debido a fugas de conductores.

Finalmente, se concluye que los resultados de probabilidad de la fatalidad relacionada con aliento alcohólico y edad, deben tomarse también con reserva debido a las fugas que impiden registrar factores sociodemográficos de los conductores. 


\section{Bibliografía}

» Aristizábal, A., Palacios, L., Milena, N. y Mesa, D. (2018). Factores que inciden en la conducta de los peatones frente a las normas de seguridad vial. Revista CIES, 9(1), 32-47.

" Baker, S., Robertson, L. y O’Neill, B. (1974). Fatal pedestrian collisions: driver negligence. American journal of public health, 64(4), 318-325.

» Berjisian, E., y Habibian, M. (2019). Developing a pedestrian destination choice model using the stratified importance sampling method. Journal of Transport Geography, 77, 39-47.

»Bliss, T. y Breen, J. (2009). Implementing the Recommendations of the World Report on Road Traffic Injury Prevention. Washington, D.C.: The World Bank Global Road Safety Facility.

»Bliss, T. (2004). Poniendo en práctica las recomendaciones del Informe Mundial sobre la Prevención de Lesiones Causadas por Accidentes de Tránsito (versión en español). Nota de Transporte No. TN-1. Washington D.C.: The World Bank.

» Cameron, C. y P. Trivedi. (2013). Regression analysis of count data. U.S.A.: CUP.

»Canitez, F. (2019). Pathways to sustainable urban mobility in developing megacities: A socio-technical transition perspective. Technological Forecasting and Social Change, 141, 319-329.

" Cantillo, V., Garcés, P. y Márquez, L. (2016). Factors influencing the occurrence of traffic accidents in urban roads: A combined GIS-Empirical Bayesian approach. DYNA, 83(195), 21-28.

"Carlotta, K. y Bertolini, L. (2017). Urban streets: Epitomes of planning challenges and opportunities at the interface of public space and mobility. Cities, 68, 48-55.

"Chen, P., Wu, C., y Zhu, S. (2016). Interaction between vehicles and pedestrians at uncontrolled mid-block crosswalks. Safety Science, 82, 68-76.

" CONAGUA (2018). Resúmenes Mensuales de Temperaturas y Lluvia. CDMX: CONAGUA. Recuperado de https://smn.cna.gob.mx/es/climatologia/ temperaturas-y-Iluvias/resumenes-mensuales-de-temperaturas-y-Iluvias (08/02/2018).

"Dada, M., Zuidgeest, M. y Hess, S. (2019). Modelling pedestrian crossing choice on Cape Town's freeways: Caught between a rock and a hard place? Transportation Research Part F: Traffic Psychology and Behavior, 60, 245-261.

»Dovey, K. y Pafka, E. (2019). What is walkability? The urban DMA. Urban Studies, 57(1), 93-108.

»Elvik, R. (2006). Laws of accident causation. Accident Analysis \& Prevention, 38(4), 742-747.

»Espinosa, I. (2013). Distancias caminables. CDMX: Trillas.

» Fávero, L. P., y Belfiore, P. (2019). Data Science for Business and Decision Making. London: Academic Press

» Forsyth, A. (2015). What is a walkable place? The walkability debate in urban design. Urban Design International, 20(4), 274-292.

"Gehl, J. (2016). Ciudades para la gente. España: El equilibrista.

" Guerrero, T. (2015). Modelo de frecuencia de accidentes en motocicletas en el 
casco urbano del municipio de Ocaña. INGENIO UFPSO, 8, 9-17.

" Gopalakrishnan, S. (2012). A public health perspective of road traffic accidents. Journal of Family Medicine and Primary Care. 1(2):144-50.

"Greene, W. (2008). Functional forms for the negative binomial model for count data. Economics Letters, 99, 585-590.

"Hall, M., Ram, Y.yShoval, N. (2018). Introduction. Walking-more than pedestrian. En M. Hall, Y. Ram, y N. Shoval (Eds.). The Routledge International Handbook of Walking. (27-36). New York: Taylor \& Francis.

» Haule, H., Sando, T., Kitali, A. y Richardson, R. (2019). Investigating proximity of crash locations to aging pedestrian residences. Accident Analysis \& Prevention, $122,215-225$.

" Helmer, T. (2015). Development of a methodology for the evaluation of Active Safety using the example of preventive pedestrian protection. Tesis Doctoral de Technische Universität Berlin, Germany.

»Hernández-Hernández, V., y De Haro-De León, L. (2014). La relación entre la centralidad urbana y los atropellamientos en Ciudad Juárez, México. Revista Hacia la Promoción de la Salud, 19(2), 81-94.

» Hernández-Vásquez, A.; Azañedo, D., Bendezú-Quispe, Guido., PachecoMendoza, J. y Chaparro, M. (2016). Sistemas de información geográfica: aplicación práctica para el estudio de atropellos en el cercado de Lima, Perú. Revista Peruana de Medicina Experimental y Salud Pública, 33(4), 725-731.

» Hilbe, J. (2011). Negative Binomial Regression. U.S.A.: CUP.

» Hilbe, J. (2014). Modeling count data. U.S.A.: CUP.

» Hijar, M. (2000). Utilidad del análisis geográfico en el estudio de las muertes por atropellamiento. Salud Pública de México, 42(3), 188-193.

»Hinojosa, R., Garrocho C., Campos, J. y Campero, A. (2015). Pronóstico de accidentes viales en el espacio intrametropolitano de Toluca: un enfoque Bayesiano. Transportes, 23(2), 43-55.

» Hoffman, J. (2019). Basic Biostatistics for Medical and Biomedical Practitioners. London: AP.

» Hosmer, D., Lemeshow, S. y Sturdivant, R. (2013). Applied logistics regression. NJ: Wiley.

» IIP. (2012). Diagnóstico de movilidad en Ciudad de México: El impacto del crecimiento vehicular (problemas, estadísticas y evaluación de políticas). Ciudad de México: Asamblea Legislativa, VII Legislatura.

» INEGI (2016). Síntesis metodológica de la estadística de accidentes de tránsito terrestre en zonas urbanas y suburbanas. Aguascalientes: INEGI.

" INEGI (2017). Anuario estadístico y geográfico de la Ciudad de México 2017. Aguascalientes: INEGI.

" INEGI. (2019a). Cuéntame. Información por entidad. Aguascalientes. Recuperado de http://cuentame.inegi.org.mx/monografias/default.aspx?tema=me (08/07/2019)

" INEGI. (2019b). Ciudad de México. Mapas para imprimir. Recuperado de http:// cuentame.inegi.org.mx/mapas/df.aspx?tema=M (o8/07/2019).

»INEGI. (2019C). Número de Solicitud CAS-11118-Y8R5Z7 CRM:0874347. Consulta 
realizada a través del Chat Oficial de INEGI el 05/07/2019 y respuesta recibida por correo electrónico el 18/07/2019.

"INEGI. (2020). Accidentes de tránsito terrestre en zonas urbanas y suburbanas. Microdatos. Aguascalientes: INEGl. Recuperado de https://www.inegi.org.mx/ programas/accidentes/default.html\#Microdatos (29/05/2020).

"Isaksson-Hellman, I. (2012). A study of bicycle and passenger car collisions based on insurance claims data. Annals of advances in automotive medicine. Association for the Advancement of Automotive Medicine. Annual Scientific Conference, 56, 3-12.

" Jacobs, J. (2011). Muerte y vida en las grandes ciudades. Salamanca: Capitán Swing.

» Jiménez, D. (2010). Comportamiento peatonal. Tesis de Grado de Magíster en Ciencias de la Ingeniería. Mención Transporte. Santiago: Universidad de Chile.

» Kröyer, H. (2015). Is $30 \mathrm{~km} / \mathrm{h}$ a 'safe' speed? Injury severity of pedestrians struck by a vehicle and the relation to travel speed and age. IATSS Research, 39(1), 4250.

»Lindenau, M.yBöhler-Baedeker, S. (2014). Citizen and Stakeholder Involvement: A Precondition for Sustainable Urban Mobility. Transportation Research Procedia, 4, 347-360.

" Liu, X., Rapik, M., Qin, X. y Barkan, C. (2013). Analysis of U.S. freight-train derailment severity using zero-truncated negative binomial regression and quantile regression. Accident Analysis \& Prevention, 59, 87-93.

» Lo, R. (2009). Walkability: what is it? Journal of Urbanism: International Research on Placemaking and Urban Sustainability, 2(2), 145-166.

"Loo, B. y Anderson, K. (2015). Spatial analysis methods of road traffic collisions. Florida: CRC.

»Mohan, D. (2019). Traffic safety: Rights and obligations. Accident Analysis \& Prevention, 128, 159-163.

"Mumford, L. (1981). The highway and the city. Connecticut: GPP.

»OMS. (2015). Informe sobre la situación mundial de la seguridad vial 2015. Ginebra: OMS.

» Öström, M., y Eriksson, A. (2001). Pedestrian fatalities and alcohol. Accident Analysis \& Prevention, 33(2), 173-180.

"Papadimitriou, E., Lassarre, S. y Yannis, G. (2017). Human factors of pedestrian walking and crossing behavior. Transportation Research Procedia, 25, 2002-2015.

" Patterson, I., Pegg, S. y Rabiah, W. (2018). Walking to promote increased physical activity. En M. Hall, Y. Ram, Y. y N. Shoval, N. (Eds.). The Routledge International Handbook of Walking. (274-286). New York: Taylor \& Francis.

»Pérez, R. y Gómez, L. (2014). La vía pública y las lesiones causadas por el tránsito. En M. Hijar (Ed.), Los accidentes como problema de salud pública en México. (69-16). México: Academia Nacional de Medicina.

»Quistberg, A., Koepsell, T., Boyle, L., Miranda, J., Johnston, B. y Ebela, B. (2014), Pedestrian signalization and the risk of pedestrian-motor vehicle collisions in Lima, Peru. Accident Analysis and Prevention, 70, 273-281.

» Rafiemanzelat, R., Imani M., Jalal A. (2017). City sustainability: the influence of walkability on built environments. Transportation Research Procedia, 24, 97-104. 
»Ramírez, G.y Ramírez, B. (2006). Colinealidad y mínimos cuadrados ponderados. Revista Venezolana de Análisis de Coyuntura, 12(1), 283-296.

»Rodríguez-Hernández, J., Campuzano-Rincón, J. e Híjar, M. (2011). Comparación de datos sobre mortalidad por atropellamientos en la Ciudad de México: ¿se han presentado cambios en una década? Salud Pública de México, 53(4), 320-328.

"Rodríguez, J., Hijar, M. y Villaveces, A. (2014). Auditorías viales e intervenciones para prevenir atropellamientos, Cuernavaca, México, 2010. Rev. Fac. Nac. Salud Pública, 32(3), 275-281.

"Sanz, A. (2016). Manual de movilidad peatonal. Caminar en la Ciudad. Madrid: Editorial Garceta.

»Scott, J. y Freese, J. (2014). Regression models for categorical dependent variables using Stata. Texas: Stata Press.

»Shaoman, Z. y Ying, Y. (2015). Based on prospect theory of pedestrian impact analysis. International Journal of Engineering Research and Applications, 5(7), 2831.

"Schmidt-Daffy, M. (2014). Prospect balancing theory: Bounded rationality of drivers' speed choice. Accident Analysis \& Prevention, 63, 49-64.

"Shatu, F., Yigitcanlar, T., y Bunker, J. (2019). Objective vs. subjective measures of street environments in pedestrian route choice behavior: Discrepancy and correlates of non-concordance. Transportation Research Part A: Policy and Practice, 126, 1-23.

»Shinar, D. (2017). Traffic safety and human behavior. United Kingdom: EGP.

»Shonkwiler, J. (2016). Variance of the truncated negative binomial distribution. Journal of Econometrics, 195(2), 209-210.

"Southworth, M. (2005). Designing the Walkable City. Journal of Urban Planning and Development, 131(4), 246-257.

»Speck, J. (2012). Walkable city. New York: NPP.

»Tabasso, C. (2011). Beber ante el peligro. Un aporte de $3 \mathrm{M}$ a la seguridad vial. Asunción: 3M.

"Tabasso, C. (2012). Paradigmas, teorías y modelos de la seguridad y la inseguridad vial. Madrid: Instituto Vial Iberoamericano.

» Talen, E. y Koschinsky, Julia. (2013). The Walkable Neighborhood: A Literature Review. International Journal of Sustainable Land Use and Urban Planning, 1(1), 42-63.

»Van Houten, R. (2011). Pedestrians. En B. Porter (Ed.), Handbook of Traffic Psychology. (353-373). Norfolk: AP.

"Vargas, G. y Carrillo, J. (2009). ¿Existen los derechos del peatón en México?. En G. Cisneros y F. Otero (Coord.), Régimen jurídico del urbanismo. Memoria del Primer Congreso de Derecho Administrativo Mexicano. (309-328). CDMX: Universidad Nacional Autónoma de México.

»Wang, W., Yuan, Z., Yang, Y., Yang, X. y Liu, Y. (2019) Factors influencing traffic accident frequencies on urban roads: A spatial panel time fixed effects error model. Plos One, 14(4), 1-18.

》Wanvik, P. (2009). Effects of road lighting: an analysis based on Dutch accident statistics 1987-2006. Accident Analysis and Prevention, 41(1), 123-128. 
»Wegman, F (2001). Transport safety performance indicators. Brussels: European Transport Safety Council.

"Wilde, G. (1982). The Theory of Risk Homeostasis: Implications for Safety and Health. Risk Analysis, 2(4), 209-225.

» Yang, X. (2019). Introduction to Algorithms for Data Mining and Machine Learning. London: AP.

"Zhang, G., Yau, K. y Chen, G. (2013). Risk factors associated with traffic violations and accident severity in China. Accident Analysis and Prevention, 59, 18-25.

"Zhou, B., Roshandeh, A., Zhang, S. y Ma, Z. (2016). Analysis of Factors Contributing to Hit-and-Run Crashes Involved with Improper Driving Behaviors. Procedia Engineering, 137, 554-562.

\section{Ramiro Flores-Xolocotzi / pinos42@hotmail.com}

Licenciado en Biología (Universidad Nacional Autónoma de México), Maestro en Ciencias (Posgrado Forestal del Colegio de Posgraduados), Doctor en Ciencias (Posgrado Forestal del Colegio de Posgraduados), Posdoctorado (Colegio Mexiquense). Investigador. Publico artículos en revistas de la especialidad en estudios urbanos: espacios públicos, parques urbanos, planeación urbana, recreación en parques citadinos y valoraciones económicas de bienes ambientales en ciudades). 